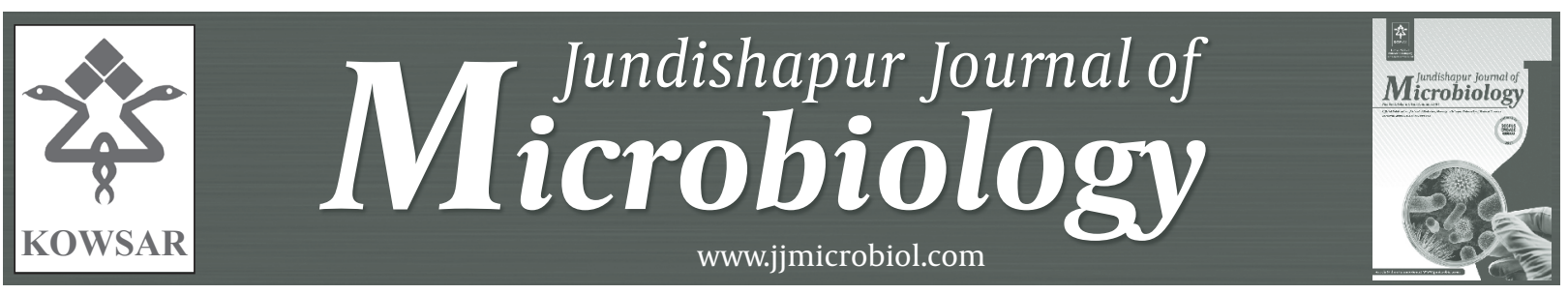

\title{
An Overview on the Global Frequency of Superficial/Cutaneous Mycoses and Deep Mycoses
}

\author{
Abdolhassan Kazemi ${ }^{1, *}$ \\ ${ }^{1}$ Tropical and Infectious Diseases Research Center, Tabriz University of Medical Science, Tabriz, IR Iran
}

*Corresponding author: Abdolhassan Kazemi, Tropical and Infectious Diseases Research Center, Tabriz University of Medical Science, Tabriz, IR Iran. Tel.: +98-4113373745, Fax:+98-4113373745, E-mail: Kazemi1338@Gmail.com.

Keywords: Mycoses; Global Frequency; Pattern Change; Health Implication

Article type: Editorial; Received: 01 Feb 2013, Revised: 09 Feb 2013, Accepted: 10 Feb 2013; DOI: 10.5812/jjm.10725

\Implication for health policy/practice/research/medical education:

The suggestions and ideas of this scientific document can be useful for clinicians, medical mycologists and public health managers for better consideration of mycoses problems globally and also locally.

Please cite this paper as:

Kazemi A. An Overview on the Global Frequency of Superficial/Cutaneous Mycoses and Deep Mycoses. Jundishapour J Microbiol. 2013:6(3): 202-4. DOI:10.5812/jjm.10725

Copyright (C) 2013, Ahvaz Jundishapur University of Medical Sciences; Published by Kowsar Corp.

This is an Open Access article distributed under the terms of the Creative Commons Attribution License (http://creativecommons.org/licenses/by/3.0), which permits unrestricted use, distribution, and reproduction in any medium, provided the original work is properly cited. 
Mycoses are classified as fungal infections caused by eumycotic organisms such as opportunistic and also pathogenic Mycoses are classified as fungal infections caused by eumycotic organisms such as opportunistic and also pathogenic Dermatophytes spp., Candida spp., Aspergillus spp., Zygomycetes spp., Feomycetes spp., Cryptococcus and some other fungal species. The frequency of opportunistic, deep and also subcutaneous mycoses compared with both the superficial and cutaneous mycoses amongst the reported cases during the last few decades increased noticeably due to the relation of deep mycoses to underlying conditions such as chemotherapy, radiotherapy, blood and solid organ cancer, hematopoietic system diseases, using a wide spectrum antibiotic, HIV+ and AIDS, endocrine abnormality, organ transplantation and etc (1).

Albeit it should be mentioned that among both the superficial and cutaneous mycoses, tinea pedis and tinea unguium increased slightly during the above period. On the contrary, tinea capitis (all three types including ecthotrix, endothrix and favous types), mannum, corporis and cruris showed a notable decreasing tendency but the Albeit it should be mentioned that among both the superficial and cutaneous mycoses, tinea pedis and tinea unguium increased slightly during the above period. On the contrary, tinea capitis (all three types including ecthotrix, endothrix and favous types), mannum, corporis and cruris showed a notable decreasing tendency but the Malassezia infection (tinea versicolor) showed no significant change (1). According to the reports and records in the last 30 years, the frequency of deep mycoses increased noticeably from $\sim 1.5 \%$ to a peak of $\sim 4.7 \%$ among suspected cases (2).

In fact, $\sim 20 \%$ of HIV+ and AIDS patients have been recorded to have an opportunistic fungal infection as one complication. Even now, cases of severe deep mycoses are severely uncontrollable, and their numbers are still increasing $(3,4)$. In agreement between clinicians and medical mycologists and according to the published papers on the trends of mycoses, over the past few decades, an increase of deep mycoses (however, the frequency of some deep mycoses decreased slowly), which include aspergillosis, zygomycosis, fusariosis, and non-Candida albicans candidiasis have been observed $(3,4)$ (Figure 1 ).

Albeit during the same period of time, some certain clinical types of superficial or cutaneous candidiasis have been controlled sufficiently by the use of effective anti-fungal agents such as fluconazole and itraconazole; however, azole-resistant $C$. albicans strains and pathogenic non-C. albicans species have been postulated as an important clinical problem. Amongst the reasons for this trend, leukemia was one of the major facts underlying the deep and opportunistic mycoses, followed by solid cancers and other blood and hematopoietic system diseases.

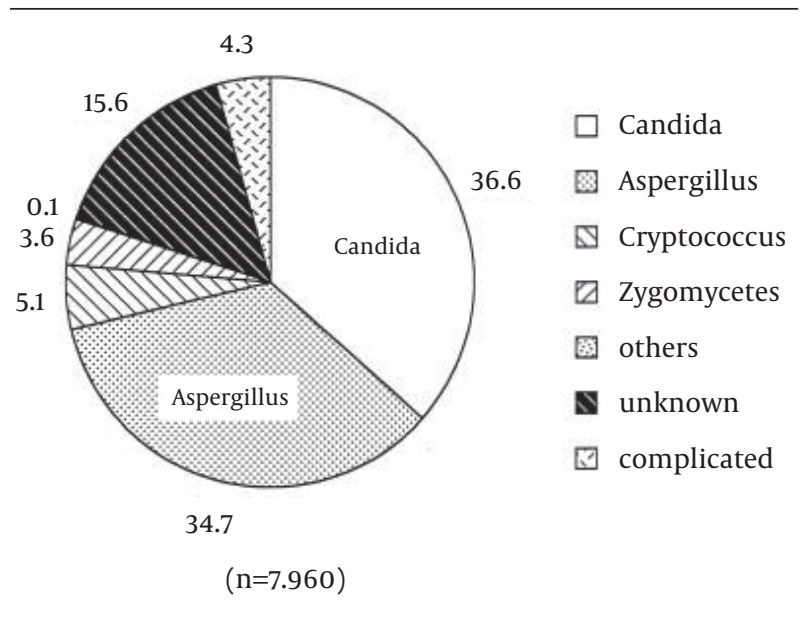

Figure 1. Causative Agents for Deep Mycoses From the Total 7,960 Cases of Mycoses

Furthermore, acute and chronic systemic aspergillosis has been increasing in bone marrow-transplanted patients (BMT), HIV+, under chemotherapy and radiotherapy and in patients with other immunocompromised conditions $(2,4,5)$.

These life treated fungal deep infections could be diagnosed and reported as:

1- The direct etiologic agent of death

2-Acute pulmonary mycosis single or both lobes of the lung

3- Acute systemic mycosis of single or more organ systems, including mycoses involving the central nervous system (CNS) (meningitis, encephalitis, meningoencephalitis)

4- Multiorgan systemic infection of more than two or three organ systems

\section{5- Fungemia}

It should also be considered that the available antifungal drugs are limited and antifungal azoles were not efficacious against acute deep mycoses such as acute bronchopulmonary aspergillosis (ABPA) and chronic bronchopulmonary aspergillosis (CBPA). It seems that the background for decrease of some opportunistic mycoses like candidiasis combined with an increase of aspergillosis (ABPA \& CBPA) or of other life treated deep mycoses could be listed as:

Using efficacious antifungal azoles worldwide such as fluconazole and itraconazole against severe deep mycoses since 1990.

A prophylactic therapy was usually given to protect immunodeficient patients from the probable acquiring of primary mycoses

Available antifungal drugs were partially efficacious for severe infections

Using effective methods for laboratory early detection (PCR, Southern \& western blotting, LAMP, Microarray) and also diagnostic techniques for both candidiasis and 
aspergillosis were inadequate and not yet fully developed.

The number of patients living longer in an immunodeficient situation increased as a result of clinical care developments $(2,3,5)$.

In immunocompromised patients the lung, bronchial system, digestive system, urogenital system and also the skin were most frequently involved regardless of the pathogen species. This suggests that the lung, bronchia, esophagus, mucocutaneous membranes, the vagina and skin are at the highest risk of being exposed to not only exogenous pathogenic fungi, such as Aspergillus, Cryptococcus, Histoplasma, Dimatiaceous fungi or Zygomycetes, but also endogenous pathogenic fungi, such as Candida species. Although Candida species are usually found commensally in the respiratory tract, digestive tract and vagina and vaginal discharge, it is probable for them to be a basic causative agent of the different types of clinical mycoses in immunodeficient patients. These facts might clarify why the esophagus, mucocutaneous membranes, lung and bronchia are found to be the organs predominantly infected by Candida. Reasonably, Candidal symptoms were also diagnosed more commonly than those of the other major opportunistic fungi in the esophagus, vagina, lung, and bronchi (3-5).

In a general perspective, an analysis of the comparison amongst frequency, distribution, epidemiology and incidence of deep mycoses and superficial/cutaneous mycoses, different occupations and leisure-time activities and health statue should be considered as well as the routes of all types of mycoses in different societies. The spread- ing of this disease in most developed countries of the world represents a considerable health problem, since it is usually accompanied by a parallel increase in the frequency of immunocompromised related diseases. In the other poorer regions of the world, deep mycoses appear primarily due to the poor healthcare systems and their diagnosis. I many cases, treatment often fail caused by the lack of efficient healthcare facilities $(2,5)$.

\section{Acknowledgements}

None declared.

\section{Financial Disclosure}

None declared.

\section{References}

1. Seebacher C, Bouchara JP, Mignon B. Updates on the epidemiology of dermatophyte infections. Mycopathologia. 2008;166(5 6):335-52

2. Lanternier F, Dannaoui E, Morizot G, Elie C, Garcia-Hermoso D, Huerre M, et al. A global analysis of mucormycosis in France: the RetroZygo Study (2005-2007). Clin Infect Dis. 2012;54 Suppl 1:S3543

3. Pettit AC, Kropski JA, Castilho JL, Schmitz JE, Rauch CA, Mobley $\mathrm{BC}$, et al. The index case for the fungal meningitis outbreak in the United States. N Engl J Med. 2012;367(22):2119-25

4. Tyczkowska-Sieron E, Bartoszko-Tyczkowska A. [Candida and Aspergillus infections in the light of a new list of alarm factors on the example of the Lodz Medical University Hospital No. 1]. Med Dosw Mikrobiol. 2012;64(3):245-53

5. Yamazaki T, Kume H, Murase S, Yamashita E, Arisawa M. Epidemiology of visceral mycoses: analysis of data in annual of the pathological autopsy cases in Japan. J Clin Microbiol. 1999;37(6):1732-8 